\title{
Habitat heterogeneity and diversity of bryophytes in
} campos rupestres

\author{
Emilia de Brito Valente ${ }^{\star}$, Kátia Cavalcanti Pôrto ${ }^{2}$ and Cid José Passos Bastos ${ }^{3}$
}

Received: December 16, 2016

Accepted: March 10, 2017

\begin{abstract}
Campos rupestres (rupestrian grasslands) are characterized by the presence of rock outcrops associated with an herbaceous-shrub physiognomy typically growing on quartzitic soils; they occur a wide variety of habitats. Bryophytes respond rapidly and efficiently to variation in microclimate. The present work aimed to study bryophyte species richness, diversity and composition, their life forms, and the substrates they colonize in exposed and shaded habitats in campos rupestres of Chapada Diamantina. Collections were made in 25 x 25 m sampling plots. One hundred and nine species were recorded and included a predominance of mosses (78 spp.) over liverworts (31 spp.). Most species (79\%) were restricted to one of the two types of habitat (exposed versus shaded). While the genera Campylopus, Polytrichum, Schlotheimia, Macromitrium and Syrrhopodon were prevalent in exposed habitats, Sphagnum, Lepidozia, Micropterygium, Bazzania and Odontoschisma prevailed in shaded habitats. The rupicolous community was more prominent than the other communities in both types of habitat. "Weft" was the most frequent life form in shaded areas, while "turf" predominated in exposed sites. The high number of rare, and exclusive, species illustrates the high degree of heterogeneity among bryophyte communities in campos rupestres, and demonstrates the importance of habitat heterogeneity for the high diversity.
\end{abstract}

Keywords: Chapada Diamantina, Bahia, Brazil, liverworts, mosses

\section{Introduction}

Bryophytes encompass three groups of plants - mosses, liverworts and hornworts - that share the absence of a vascular system, small size and water-dependent sexual reproduction. They usually have photosynthetic laminae (leaves) comprised of a single-cell layer and absorb nutrients and water throughout the surface of the gametophyte, and thus occur in humid and shady environments. They reach their greatest abundance and diversity in rainforests, but can be found in all types of environments from polar to arid regions including deserts; they even inhabit urban areas and industrial settings with high pollution levels (Glime 2007; Vanderpoorten \& Goffinet 2009). Variation in bryophyte species richness, diversity and composition, and their life forms, are clearly linked to microclimatic conditions, but especially to light and water availability (Mägdefrau 1982). For this reason, bryophytes are considered efficient bioindicators of environmental quality (Frahm \& Gradstein 1991).

Chapada Diamantina is a mountainous region located in the Caatinga of northeastern Brazil (IBGE 2004), and is one of the eight ecoregions recognized within this biome (Velloso et al. 2002). It exhibits a mosaic of plant formations mainly determined by topography, elevation, climate and hydrography,

\footnotetext{
${ }^{1}$ Programa de Pós-Graduação em Botânica, Departamento de Ciências Biológicas, Universidade Estadual de Feira de Santana, Av. Transnordestina, s/n, Novo Horizonte, 44036-900, Feira de Santana, BA, Brazil

${ }^{2}$ Programa de Pós-Graduação em Biologia Vegetal, Departamento de Botânica, Centro de Ciências Biológicas, Universidade Federal de Pernambuco, 50670-901, Recife, PE, Brazil

${ }^{3}$ Departamento de Botânica, Instituto de Biologia, Universidade Federal da Bahia, 40170-280, Salvador, BA, Brazil
}

* Corresponding author: ebvalente@gmail.com 
including campos rupestres (rupestrian grasslands), which is the most typical of these formations. According to Harley (1995a), campos rupestres are distributed in a manner analogous to an archipelago, with several elevated areas separated by lower areas with unique environmental characteristics.

Another striking feature of campos rupestres is the high degree of habitat heterogeneity as a result of substrate variation, proportion of exposed rock, and the presence of rock blocks and sandy sediments (Conceição 2000; 2008; Conceição \& Giulietti 2002; Conceição \& Pirani 2005; 2007). This heterogeneity facilitates high levels of diversity and endemism (Alves \& Kolbek 1993; Harley 1995a; Giulietti et al. 1996; Conceição 2000; Conceição \& Giulietti 2002; Conceição \& Pirani 2005).

Studies have indicated that the composition of the phanerogamic flora of the campos rupestres of Chapada Diamantina varies considerably, even within small geographical distances and among equally rich areas (Conceição \& Giulietti 2002; Zappi et al. 2003; Conceição \& Pirani 2005; 2007; Conceição et al. 2005).

Knowledge of the bryophytes of the campos rupestres of Chapada Diamantina is limited to species lists for Pico das Almas, a municipality of Rio de Contas (Harley 1995b) and various other municipalities in this ecoregion (Bastos et al. 2000), and a survey of mosses carried out in the municipality of Miguel Calmon (Ballejos \& Bastos 2009a; b).

Recent studies have revealed that the bryophyte flora of the Chapada Diamantina region is rich and diverse, and includes 80 $\%$ of the bryophyte taxa recorded in the state of Bahia (Valente et al. 2011), with $40 \%$ of this richness being found in campos rupestres and $51 \%$ in forests (Valente et al. 2013a; b). According to Bastos et al. (2000), the most represented families in the campos rupestres of Chapada Diamantina are Dicranaceae, Orthotrichaceae, Sphagnaceae and Leucobryaceae, among Bryophyta, and Lepidoziaceae, Plagiochilaceae, Herbertaceae and Trichocoleaceae, among Marchantiophyta. These authors also report that the most frequently encountered taxa are also restricted to Chapada Diamantina and have not been found elsewhere in the state. Bryophytes of campos rupestres have been surveyed in other regions of Brazil, including Serra do Cipó (Yano 1987) and Flora of Grão Mongol (Yano \& Peralta 2009), both in the state of Minas Gerais.

This study aimed to survey the bryophyte communities of the campos rupestres of Chapada Diamantina and describe the species richness diversity and composition, the variety of life forms and the substrates colonized in different areas and habitats throughout this region.

\section{Materials and methods}

\section{Study Area}

Chapada Diamantina is located in the northern portion of the Cadeia do Espinhaço, the main mountain chain in eastern Brazil, and extends from Serra da Jacobina (1000'S) in Bahia to Serra de Ouro Branco, near the city of Ouro Preto ( $21^{\circ} 25$ 'S), in Minas Gerais (Harley 1995a). It encompasses an area of $50,000 \mathrm{~km}^{2}$, and extends for $400 \mathrm{~km}$ northsouth and 50 to $100 \mathrm{~km}$ east-west $\left(10^{\circ} 00^{\prime}-14^{\circ} 00^{\prime} \mathrm{S} ; 40^{\circ} 10^{\prime}\right.$ $44^{\circ} 30^{\prime} \mathrm{W}$ ), with elevations ranging from 400 to 2,033 m.a.s.l. (Harley 1995a; Rocha et al. 2005).

According to Nolasco et al. (2008), meteorological and rainfall stations in the Chapada Diamantina are few and insufficient for modeling local microclimates, and are generally used for describing regional trends based on data collected over multiple years. Based on rainfall data recorded by these stations over the past 20 years, a rainy season occurs between November and April, with maximum precipitation in December (139 $\mathrm{mm}$ ), and a dry season occurs between May and October, with minimum precipitation in August (20 mm). Mean monthly rainfall exceeds $100 \mathrm{~mm}$ during the rainy season, while it averages only $35 \mathrm{~mm}$ during the dry season. Total annual precipitation varies between 600 and 1,100 mm (SEI 2003; Agritempo 2010).

Temperatures in Chapada Diamantina generally range $15-30{ }^{\circ} \mathrm{C}$, but can reach freezing $\left(0^{\circ} \mathrm{C}\right)$ in the dry season between June and August, and $30^{\circ} \mathrm{C}$ in the rainy season between December and January. Fog is typically present until 09:00 h on ordinary days and 12:00 h on rainy days, and is common throughout the region (Nolasco et al. 2008).

Campos rupestres are mainly characterized by the presence of rock outcrops associated with herbaceous-shrub physiognomy typically growing on quartzitic soils. The main plant families present are Velloziaceae, Melastomataceae, Eriocaulaceae, Xyridaceae and Orchidaceae, usually at elevations above $900 \mathrm{~m}$. Forest formations can occur in areas of campos rupestres, especially along watercourses, and numerous waterfalls and cliffs are present in these areas (Conceição et al. 2005).

\section{Sampling and study material}

Nine botanical sampling trips were made between the years 2007 and 2008. Material was collected within $25 \mathrm{x}$ $25 \mathrm{~m}$ standard plots located in 15 different areas (Frahm 1994). The study areas were distributed from north to south with eight of them being within conservation units (Tab. 1).

The botanical material was identified using Fulford (1963; 1966; 1968), Crum (1984), Yano et al. (1985), Frahm (1991), Reese (1993), Sharp et al. (1994), Buck (1998), Gradstein et al. (2001), Allen (2002), Gradstein \& Costa (2003) and Vaz-Imbassahy \& Costa (2008), among other more specialized papers. Vouchers were deposited in HUEFS and UFP, according Thiers (2017). The classification system adopted for mosses was that of Goffinet et al. (2009), while for liverworts it was that of Crandall-Stotler et al. (2009).

The areas surveyed were classified, according to their physical features, into two habitat types, exposed and shaded. Exposed habitats possess a large proportion of exposed rock surface and herbaceous and shrub vegetation. 
Table 1. Characterization of campo rupestre areas of Chapada Diamantina, Bahia, Brasil. PNCD - Parque Nacional da Chapada Diamantina; MCFD Monumento Cachoeira do Ferro Doido; APA-SB - APA Serra do Barbado. E1 - E8 = habitat exposed areas; S1 - S7 = habitat shaded areas.

\begin{tabular}{|c|c|c|c|c|}
\hline Areas & Municipality & Conservation unit & $\begin{array}{c}\text { Altitude } \\
\text { Cm.a.s.I.J }\end{array}$ & \begin{tabular}{c} 
Geographic coordinates \\
\hline E1
\end{tabular} Piatã $^{\text {E2 }}$ \\
\hline E3 & Piatã & - & 1,286 & $13^{\circ} 09^{\prime} 01.2^{\prime \prime} \mathrm{S}-41^{\circ} 45^{\prime} 38.7^{\prime \prime} \mathrm{W}$ \\
\hline E4 & Piatã & - & 1,479 & $13^{\circ} 08^{\prime} 57.8^{\prime \prime} \mathrm{S}-41^{\circ} 45^{\prime} 31.7^{\prime \prime} \mathrm{W}$ \\
\hline E5 & Abaíra & - & 1,665 & $13^{\circ} 09^{\prime} 05.3^{\prime \prime} \mathrm{S}-41^{\circ} 45^{\prime} 07.4^{\prime \prime} \mathrm{W}$ \\
\hline E6 & Abaíra & APA-SB & 1,750 & $13^{\circ} 17^{\prime} 28.6^{\prime \prime} \mathrm{S}-41^{\circ} 54^{\prime} 12.1^{\prime \prime} \mathrm{W}$ \\
\hline E7 & APA-SB & 1,850 & $13^{\circ} 17^{\prime} \mathrm{S}-41^{\circ} 54^{\prime} \mathrm{W}$ \\
\hline E8 & Abaíra & APA-SB & 2,037 & $13^{\circ} 17^{\prime} 47.9^{\prime \prime} \mathrm{S}-41^{\circ} 54^{\prime} 26.8^{\prime \prime} \mathrm{W}$ \\
\hline S1 & Palmeiras & APA-M & 1,150 & $12^{\circ} 27^{\prime} \mathrm{S}-41^{\circ} 28^{\prime} \mathrm{W}$ \\
\hline S2 & Palmeiras & APA-M & 1,170 & $12^{\circ} 27^{\prime} \mathrm{S}-41^{\circ} 28^{\prime} \mathrm{W}$ \\
\hline S3 & Moro do Chapéu & MCFD & 1,000 & $11^{\circ} 37^{\prime} 30.0^{\prime \prime} \mathrm{S}-40^{\circ} 59^{\prime} 58.6^{\prime \prime} \mathrm{W}$ \\
\hline S4 & Lençóis & - & 722 & $12^{\circ} 27^{\prime} 42.6^{\prime \prime} \mathrm{S}-41^{\circ} 25^{\prime} 04.9^{\prime \prime} \mathrm{W}$ \\
\hline S5 & Lençóis & - & 720 & $12^{\circ} 27^{\prime} 42.8^{\prime \prime} \mathrm{S}-41^{\circ} 25^{\prime} 03.7^{\prime \prime} \mathrm{W}$ \\
\hline S6 & Lençóis & PNCD & $12^{\circ} 27^{\prime} \mathrm{S}-41^{\circ} 28^{\prime} \mathrm{W}$ \\
\hline S7 & Piatã & - & 800 & $13^{\circ} 05^{\prime} 10.2^{\prime \prime} \mathrm{S}-41^{\circ} 51^{\prime} 12.4^{\prime \prime} \mathrm{W}$ \\
\hline
\end{tabular}

They correspond to areas of campos rupestres, including primarily the habitats described by Conceição \& Pirani (2005) as "rocky outcrops" and smaller proportions of "ditches" and "insets". Shaded areas possess rock cracks near waterfalls or rivers and large "ditch" areas with a predominance of trees. They correspond to small caves in waterfalls and resemble the habitat described by Alves \& Kolbek (1993) in campos rupestres of the southern portion of Cadeia do Espinhaço, except for its association with cascades and rivers.

The 15 study areas were coded according to the initial letter of the habitat type, that is, exposed (E1 to E8) and shaded (S1 to S7) habitats. Among shaded areas, S1 to S5 corresponded to cracks in rocks near waterfalls, while $S 6$ and S7 correspond to large blocks of rock with trees and shrubs.

Classification of the life form of the bryophytes was based on Mägdefrau (1982), Richards (1984) and Bates (1998). This parameter refers to the manner in which individuals are assembled, which involves branching pattern, growth direction and modifications reflecting habitat conditions Thus, life forms are influenced by the environment.

The frequency of occurrence for each species was classified as: rare (up to three occurrences throughout the study areas), occasional (between four and 10 occurrences) and frequent (> 10 occurrences).

\section{Data analysis}

In order to verify sampling sufficiency, species richness was estimated with Chao's equation (1984) using EstimateS software (Cowell 2006). Species richness and diversity were calculated for each study area. The Shannon-Wiener (H ) index with natural logarithm (Napierian base) was used for calculating diversity. In this case, the frequency of each species (number of samples of the species collected in the area) instead of abundance (total number of individuals of each species collected in the area) was used in the calculations because the small size and fragmented nature of bryophytes prevent quantification of individuals (Bates 1982). Floristic composition of the study areas was compared based on the presence and absence of species using the Bray-Curtis method. Diversity and similarity were analyzed in PRIMER 5.1 software (Clarke \& Warwick 1994).

Species richness of the study areas was compared using the T-test while normality was tested using the KolmogorovSmirnov test. The level of significance adopted was $\mathrm{p}>0.05$. Both tests were performed using BioEstat 2.0 software (Ayres et al. 2000).

\section{Results}

\section{Richness, diversity and composition of study areas}

One hundred and nine species (Tab. 2) were recorded in the study, representing $79 \%$ of the total richness estimated for the campos rupestres of Chapada Diamantina according to Chao's estimator. The mean diversity for the areas was $\mathrm{H}^{\prime}=2.33$ and the total diversity was $\mathrm{H}^{\prime}=4.13$ (Tab. 3 ).

There was a predominance of mosses (72 spp.) over liverworts (37 spp.). The richest moss families were Leucobryaceae (15 spp.), Sematophyllaceae (12 spp.), Orthotrichaceae (nine spp.) and Sphagnaceae (seven spp.), while the richest liverwort families were Lepidoziaceae (nine spp.) and Lejeuneaceae (five spp.). Floristic composition differed considerably among areas, although there was one cluster of seven of the eight exposed areas, while four of the seven shaded areas formed a cluster (Fig. 1). However, similarities among areas were low, even among the exposed 
Table 2. Bryophytes species found at campo rupestre areas studies in the Chapada Diamantina, Bahia, Brazil. Frequecy of species in the habitat $\mathrm{E}$ Exposed, S - Shaded, T -Total frequency; C = community by substrate: Ru - Rupicolous, Te - Terricolous, Co - Corticicolous, Ex - Epixylous; L.F. = life forms: TF - turf, $\mathrm{W}$ - weft, TL - thallose, $\mathrm{M}$ - mat, $\mathrm{P}$ - pendant.

\begin{tabular}{|c|c|c|c|c|c|c|}
\hline Taxa & $\mathbf{E}$ & S & $\mathbf{T}$ & C & L.F. & Voucher \\
\hline Acroporium caespitosum (Hedw.) W.R. Buck & 0 & 1 & 1 & $\mathrm{Ru}$ & W & Valente, E.B. 473 \\
\hline Anastrophyllum piligerum (Reinw., Blume \& Nees) Steph. & 1 & 0 & 1 & $\mathrm{Ru}$ & W & Valente, E.B. 795 \\
\hline Anoplolejeunea conferta (C.F.W. Meissn. ex Spreng.) A. Evans & 9 & 0 & 9 & $\mathrm{Ru}$ & W & Valente, E.B. 753 \\
\hline Aptychopsis pyrrhophylla (Müll. Hal.) Wijk \& Margad. & 0 & 2 & 2 & $\mathrm{Ru}$ & W & Valente, E.B. 1535 \\
\hline Bazzania falcata (Lindenb.) Trevis. & 2 & 0 & 2 & $\mathrm{Ru}$ & W & Valente, E.B. 770 \\
\hline Bazzania heterostipa (Steph.) Fulford & 4 & 0 & 4 & $\mathrm{Ru}$ & W & Valente, E.B. 715 \\
\hline Bazzania nitida (F. Weber) Grolle & 1 & 0 & 1 & $\mathrm{Ru}$ & $\mathrm{W}$ & Valente, E.B. 750 \\
\hline Bryum argeteum Hedw. & 3 & 0 & 3 & $\mathrm{Ru}$ & $\mathrm{Tf}$ & Valente, E.B. 740 \\
\hline Callicostella merkelii (Hornsch.) A. Jaeger & 0 & 4 & 4 & $\mathrm{Ru}$ & W & Valente, E.B. 852 \\
\hline Callicostella pallida (Hornsch.) Ångstr. & 0 & 2 & 2 & $\mathrm{Ru}$ & W & Valente, E.B. 884 \\
\hline Calymperes palisotii Schwägr. & 0 & 1 & 1 & $\mathrm{Ru}$ & $\mathrm{tf}$ & Valente, E.B. 447 \\
\hline Calypogeia laxa Lindenb. \& Gottsche & 0 & 1 & 1 & $\mathrm{Ru}$ & W & Valente, E.B. 638 \\
\hline Campylopus arctocarpus (Hornsch.) Mitt. & 11 & 3 & 14 & $\mathrm{Ru}, \mathrm{Te}$ & Tf & Valente, E.B. 705 \\
\hline Campylopus heterostachys (Hampe) A. Jaeger & 2 & 3 & 5 & $\mathrm{Ru}$ & Tf & Valente, E.B. 942 \\
\hline Campylopus julaceus A. Jaeger & 11 & 2 & 13 & $\mathrm{Ru}, \mathrm{Te}$ & $\mathrm{Tf}$ & Valente, E.B. 661 \\
\hline Campylopus filifolius var. longifolius (E.B. Bartram) E.B. Bartram & 0 & 1 & 1 & $\mathrm{Ru}$ & $\mathrm{Tf}$ & Valente, E.B. 518 \\
\hline Campylopus fragilis (Brid.) Bruch \& Schimp. & 1 & 0 & 1 & $\mathrm{Ru}$ & $\mathrm{Tf}$ & Valente, E.B. 829 \\
\hline Campylopus occultus Mitt. & 1 & 2 & 3 & $\mathrm{Ru}$ & Tf & Valente, E.B. 717 \\
\hline Campylopus pilifer Brid. & 45 & 4 & 49 & $\mathrm{Ru}, \mathrm{Te}$ & $\mathrm{Tf}$ & Valente, E.B. 656 \\
\hline Campylopus savannarum (Müll. Hal.) Mitt. & 1 & 2 & 3 & $\mathrm{Ru}, \mathrm{Te}$ & Tf & Valente, E.B. 732 \\
\hline Campylopus subcuspidatus (Hampe) A. Jaeger & 8 & 0 & 8 & $\mathrm{Ru}$ & Tf & Valente, E.B. 1501 \\
\hline Campylopus uleanus (Müll. Hal.) Broth. & 1 & 0 & 1 & $\mathrm{Ru}$ & $\mathrm{tf}$ & Valente, E.B. 696 \\
\hline Campylopus widgrenii (Müll. Hal.) Mitt. & 0 & 3 & 3 & $\mathrm{Ru}$ & $\mathrm{Tf}$ & Valente, E.B. 937 \\
\hline Cheilolejeunea filiformis (Sw.) W. Ye, R.L. Zhu \& Gradst. & 0 & 1 & 1 & $\mathrm{Ru}$ & W & Valente, E.B. 1247 \\
\hline Cheilolejeunea oncophylla (Ångstr.) Grolle \& E. Reiner & 4 & 0 & 4 & Co & M & Valente, E.B. 756 \\
\hline Cheilolejeunea xanthocarpa (Lehm. \& Lindenb.) Malombe & 1 & 0 & 1 & Co & M & Valente, E.B. 690 \\
\hline Chiloscyphus martianus (Nees) J.J.Engel \& R.M.Shust. & 0 & 6 & 6 & $\mathrm{Ru}$ & W & Valente, E.B. 609 \\
\hline Colobodontium vulpinum (Mont.) S.P. Churchill \& W.R. Buck & 0 & 1 & 1 & $\mathrm{Ru}$ & Tf & Valente, E.B. 622 \\
\hline Dicranodontium pulchroalare subsp. brasiliense (Herzog) J.-P. Frahm & 0 & 1 & 1 & $\mathrm{Ru}$ & Tf & Valente, E.B. 642 \\
\hline Drepanolejeunea anoplantha (Spruce) Steph. & 1 & 0 & 1 & Co & W & Valente, E.B. 800 \\
\hline Entodontopsis leucostega (Brid.) W.R. Buck \& Ireland & 0 & 1 & 1 & $\mathrm{Ru}$ & M & Valente, E.B. 950 \\
\hline Entodon cf. macropodus (Hedw.) Müll. Hal. & 0 & 1 & 1 & $\mathrm{Ru}$ & M & Valente, E.B. 530 \\
\hline Fabronia macroblepharis Schwägr. & 1 & 0 & 1 & Co & M & Valente, E.B. 815 \\
\hline Frullania atrata (Sw.) Dumort. & 0 & 2 & 2 & $\mathrm{Ru}$ & W & Valente, E.B. 1248 \\
\hline Frullania brasiliensis Raddi & 7 & 0 & 7 & $\mathrm{Ru}$ & W & Valente, E.B. 1504 \\
\hline Frullania kunzei Lehm. \& Lindenb. & 2 & 1 & 3 & $\mathrm{Ru}$ & M & Valente, E.B. 735 \\
\hline Frullania riojaneirensis (Raddi) Spruce & 0 & 1 & 1 & $\mathrm{Ru}$ & M & Valente, E.B. 933 \\
\hline Frullania setigera Steph. & 0 & 3 & 3 & Co & W & Valente, E.B. 1262 \\
\hline Funaria hygrometrica Hedw. & 6 & 0 & 6 & $\mathrm{Ru}, \mathrm{Te}, \mathrm{Co}$ & Tf & Valente, E.B. 667 \\
\hline Gemmabryum radiculosum (Brid.) J.R. Spence \& H.P. Ramsay & 1 & 0 & 1 & $\mathrm{Ru}$ & tf & Valente, E.B. 726 \\
\hline Holomitrium arboreum Mitt. & 2 & 0 & 2 & $\mathrm{Ru}$ & Tf & Valente, E.B. 1506 \\
\hline Holomitrium olfersianum Hornsch. & 3 & 4 & 7 & $\mathrm{Ru}$ & Tf & Valente, E.B. 1487 \\
\hline Isopterygium jamaicense (E.B. Bartram) W.R. Buck & 0 & 3 & 2 & $\mathrm{Te}$ & M & Valente, E.B. 626 \\
\hline Isopterygium tenerifolium Mitt. & 0 & 2 & 2 & $\mathrm{Ru}, \mathrm{Te}$ & M & Valente, E.B. 629 \\
\hline Isopterygium tenerum (Sw.) Mitt. & 0 & 1 & 1 & $\mathrm{Ru}$ & M & Valente, E.B. 1204 \\
\hline Kurzia capillaris (Sw.) Grolle & 2 & 6 & 8 & $\mathrm{Ru}$ & W & Valente, E.B. 752 \\
\hline Lepidozia cupressina (Sw.) Lindenb. & 8 & 1 & 9 & $\mathrm{Ru}, \mathrm{Te}$ & W & Valente, E.B. 750 \\
\hline Lepidozia inaequalis Lehm. \& Lindenb. & 2 & 0 & 2 & $\mathrm{Ru}$ & W & Valente, E.B. 794 \\
\hline Leptoscyphus amphibolius (Nees) Grolle & 1 & 0 & 1 & $\mathrm{Ru}$ & W & Valente, E.B. 1471 \\
\hline Leucobryum albidum (Brid. ex P. Beauv.) Lindb. & 2 & 0 & 2 & $\mathrm{Ru}$ & Tf & Valente, E.B. 810 \\
\hline Leucobryum crispum Müll. Hal. & 2 & 5 & 7 & $\mathrm{Ru}$ & Tf & Valente, E.B. 1549 \\
\hline Leucobryum giganteum Müll. Hal. & 0 & 5 & 5 & $\mathrm{Te}$ & Tf & Valente, E.B. 1315 \\
\hline Leucobryum martianum (Hornsch.) Hampe ex Müll. Hal. & 0 & 8 & 9 & $\mathrm{Ru}, \mathrm{Te}, \mathrm{Co}$ & Tf & Valente, E.B. 871 \\
\hline Lophocholea mandonii Steph. & 0 & 2 & 2 & Ex & W & Valente, E.B. 644 \\
\hline
\end{tabular}


Table 2. Cont

\begin{tabular}{|c|c|c|c|c|c|c|}
\hline Taxa & E & $\mathbf{S}$ & $\mathbf{T}$ & C & L.F. & Voucher \\
\hline Macrocoma cf. gastonyi D.H. Norris \& Vitt & 0 & 1 & 1 & $\mathrm{Ru}$ & $\mathrm{Tf}$ & Valente, E.B. 1291 \\
\hline Macromitrium cirrosum (Hedw.) Brid. & 0 & 1 & 1 & Co & Tf & Valente, E.B. 1300 \\
\hline Macromitrium cf. longifolium (Hook.) Brid. & 0 & 1 & 1 & $\mathrm{Ru}$ & $\mathrm{tf}$ & Valente, E.B. 1255 \\
\hline Macromitrium microstomum (Hook. \& Grev.) Schwägr. & 0 & 3 & 3 & Co & Tf & Valente, E.B. 1319 \\
\hline Macromitrium punctatum (Hook. \& Grev.) Brid. & 1 & 0 & 1 & $\mathrm{Ru}$ & Tf & Valente, E.B. 1466 \\
\hline Metzgeria decipiens (C. Massal.) Schiffn. & 1 & 0 & 1 & $\mathrm{Ru}$ & $\mathrm{Tl}$ & Valente, E.B. 706 \\
\hline Micropterygium campanense Spruce ex Reimers & 0 & 2 & 2 & $\mathrm{Ru}, \mathrm{Te}$ & W & Valente, E.B. 862 \\
\hline Micropterygium reimersianum Herzog & 5 & 2 & 7 & $\mathrm{Ru}$ & W & Valente, E.B. 765 \\
\hline Neesioscyphus homophyllus (Nees) Grolle & 0 & 1 & 1 & $\mathrm{Ru}$ & W & Valente, E.B. 1394 \\
\hline Octoblepharum albidum Hedw. & 3 & 9 & 12 & $\mathrm{Ru}$ & Tf & Valente, E.B. 725 \\
\hline Octoblepharum cocuiense Mitt. & 1 & 2 & 3 & $\mathrm{Ru}$ & Tf & Valente, E.B. 773 \\
\hline Odontoschisma brasiliense Steph. & 4 & 0 & 4 & $\mathrm{Ru}$ & W & Valente, E.B. 671 \\
\hline Odontoschisma denudatum (Nees) Dumort. & 5 & 1 & 6 & $\mathrm{Ru}$ & W & Valente, E.B. 751 \\
\hline Odontoschisma falcifolium Steph. & 1 & 2 & 2 & $\mathrm{Ru}, \mathrm{Te}$ & W & Valente, E.B. 470 \\
\hline Odontoschisma longiflorum Steph. & 0 & 1 & 1 & $\mathrm{Ru}$ & W & Valente, E.B. 456 \\
\hline Orthostichopsis praetermissa W.R.Buck & 0 & 6 & 6 & $\mathrm{Co}, \mathrm{Ru}, \mathrm{Te}$ & $\mathrm{P}$ & Valente, E.B. 1272 \\
\hline Pallavicinia lyellii (Hook.) Gray & 0 & 1 & 1 & $\mathrm{Ru}$ & $\mathrm{Tl}$ & Valente, E.B. 489 \\
\hline Philonotis cernua (Wilson) D.G. Griffin \& W.R. Buck & 0 & 1 & 1 & $\mathrm{Ru}$ & Tf & Valente, E.B. 613 \\
\hline Philonotis hastata (Duby) Wijk \& Margad. & 0 & 2 & 2 & $\mathrm{Ru}$ & $\mathrm{Tf}$ & Valente, E.B. 630 \\
\hline Plagiochila corrugata (Nees) Nees \& Mont. & 1 & 3 & 4 & Co & W & Valente, E.B. 1283 \\
\hline Plagiochila fragilis Taylor & 3 & 0 & 3 & $\mathrm{Ru}$ & W & Valente, E.B. 780 \\
\hline Plagiochila simplex (Sw.) Lindenb. & 1 & 1 & 2 & $\mathrm{Ru}$ & W & Valente, E.B. 507 \\
\hline Polytrichum juniperinum Hedw. & 15 & 0 & 15 & $\mathrm{Ru}$ & W & Valente, E.B. 659 \\
\hline Pterogonidium cf. pulchellum (Hook.) Müll. Hal. & 0 & 1 & 1 & Ex & M & Valente, E.B. 1302 \\
\hline Pyrrhobryum spiniforme (Hedw.) Mitt. & 0 & 3 & 3 & $\mathrm{Ru}, \mathrm{Ex}$ & tf & Valente, E.B. 1242 \\
\hline Rhacocarpus purpurascens (Brid.) Paris & 6 & 0 & 6 & $\mathrm{Ru}, \mathrm{Te}$ & W & Valente, E.B. 1467 \\
\hline Rhacopilopsis trinitensis (Müll. Hal.) E. Britton \& Dixon & 0 & 1 & 1 & $\mathrm{Ru}$ & M & Valente, E.B. 522 \\
\hline Riccardia sp. & 0 & 1 & 1 & $\mathrm{Ru}$ & $\mathrm{Tl}$ & Valente, E.B. 649 \\
\hline Schlotheimia jamesonii (Arn.) Brid. & 0 & 3 & 3 & $\mathrm{Ru}$ & Tf & Valente, E.B. 529 \\
\hline Schlotheimia rugifolia (Hook.) Schwägr. & 10 & 15 & 25 & Co, $\mathrm{Ex}, \mathrm{Ru}$ & Tf & Valente, E.B. 698 \\
\hline Schlotheimia torquata (Sw. ex Hedw.) Brid. & 0 & 1 & 1 & $\mathrm{Ru}$ & Tf & Valente, E.B. 450 \\
\hline Schlotheimia trichomitria Schwägr. & 0 & 4 & 4 & $\mathrm{Co}, \mathrm{Ru}$ & Tf & Valente, E.B. 1249 \\
\hline Sematophyllum adnatum (Michx.) E. Britton & 1 & 4 & 5 & $\mathrm{Ru}$ & W & Valente, E.B. 1304 \\
\hline Sematophyllum galipense (Müll. Hal.) Mitt. & 0 & 2 & 2 & $\mathrm{Ru}$ & W & Valente, E.B. 637 \\
\hline Sematophyllum subpinnatum (Brid.) E. Britton & 0 & 2 & 2 & $\mathrm{Ru}$ & W & Valente, E.B. 934 \\
\hline Sematophyllum subsimplex (Hedw.) Mitt. & 0 & 5 & 5 & $\mathrm{Ru}$ & W & Valente, E.B. 435 \\
\hline Sematophyllum sp. & 1 & 4 & 5 & $\mathrm{Ru}$ & W & Valente, E.B. 908 \\
\hline Sphagnum capillifolium var. capillifolium (Ehrh.) Hedw. & 0 & 5 & 5 & $\mathrm{Ru}, \mathrm{Te}$ & Tf & Valente, E.B. 931 \\
\hline Sphagnum erythrocalyx Hampe & 5 & 1 & 6 & $\mathrm{Ru}, \mathrm{Te}$ & Tf & Valente, E.B. 461 \\
\hline Sphagnum subsecundum var. rufescens (Nees \& Hornsch.) Hueb. & 0 & 4 & 4 & $\mathrm{Ru}$ & tf & Valente, E.B. 854 \\
\hline Sphagnum longistolo Müll. Hal. & 2 & 0 & 2 & $\mathrm{Ru}$ & Tf & Valente, E.B. 1468 \\
\hline Sphagnum oxyphyllum Warnst. & 1 & 1 & 2 & $\mathrm{Ru}$ & Tf & Valente, E.B. 1572 \\
\hline Sphagnum palustre L. & 2 & 7 & 9 & $\mathrm{Ru}, \mathrm{Te}$ & Tf & Valente, E.B. 1484 \\
\hline Sphagnum recurvum P. Beauv. & 0 & 1 & 1 & $\mathrm{Ru}$ & Tf & Valente, E.B. 615 \\
\hline Squamidium brasiliense (Hornsch.) Broth. & 0 & 4 & 4 & Co & $\mathrm{P}$ & Valente, E.B. 1280 \\
\hline Symphyogyna aspera Steph. ex MacCormick & 0 & 1 & 1 & $\mathrm{Ru}$ & $\mathrm{Tl}$ & Valente, E.B. 552 \\
\hline Symphyogyna brasiliensis Nees & 0 & 3 & 3 & $\mathrm{Ru}$ & $\mathrm{Tl}$ & Valente, E.B. 946 \\
\hline Syrrhopodon ligulatus Mont. & 0 & 2 & 2 & $\mathrm{Ru}$ & Tf & Valente, E.B. 443 \\
\hline Syrrhopodon prolifer var. prolifer Schwägr. & 11 & 23 & 34 & $\mathrm{Ex}, \mathrm{Ru}$ & Tf & Valente, E.B. 722 \\
\hline Telaranea diacantha (Mont.) J.J. Engel \& G.L. Merr. & 0 & 1 & 1 & $\mathrm{Ru}$ & $\mathrm{W}$ & Valente, E.B. 503 \\
\hline Thamniopsis undata (Hedw.) W.R. Buck & 0 & 3 & 3 & $\mathrm{Ru}$ & W & Valente, E.B. 907 \\
\hline Tortella humilis (Hedw.) Jenn. & 3 & 0 & 3 & $\mathrm{Ru}, \mathrm{Te}$ & Tf & Valente, E.B. 694 \\
\hline Trichosteleum cf. microstegium (Besch.) A. Jaeger & 0 & 1 & 1 & $\mathrm{Ru}$ & M & Valente, E.B. 543 \\
\hline Trichosteleum subdemissum (Schimp. ex Besch.) A. Jaeger & 0 & 1 & 1 & $\mathrm{Ru}$ & M & Valente, E.B. 885 \\
\hline Wijkia flagellifera (Broth.) H.A. Crum & 0 & 1 & 1 & Ex & M & Valente, E.B. 1308 \\
\hline
\end{tabular}


Table 3. Richness, sampling and diversity of bryophytes by campo rupestre area of Chapada Diamantina, Bahia, Brazil. E1 - E8 = habitat exposed areas; $\mathrm{S} 1-\mathrm{S} 7$ = habitat shaded areas; $\mathrm{S}=$ specific richness; $\mathrm{N}=$ sample; $\mathrm{H}=$ diversity.

\begin{tabular}{|c|c|c|c|c|c|c|c|c|c|c|c|c|c|c|c|c|}
\hline Áreas & E1 & E2 & E3 & E4 & E5 & E6 & E7 & E8 & S1 & S2 & S3 & S4 & S5 & S6 & S7 & T0TAL \\
\hline S & 14 & 29 & 13 & 16 & 10 & 9 & 4 & 4 & 14 & 12 & 15 & 25 & 18 & 23 & 21 & 109 \\
\hline $\mathrm{N}$ & 26 & 63 & 56 & 27 & 12 & 23 & 11 & 11 & 20 & 16 & 36 & 50 & 26 & 23 & 58 & 460 \\
\hline$H^{\prime}(\log )$ & 2.15 & 3.2 & 2.1 & 2.6 & 2.3 & 1.9 & 1.2 & 0.9 & 2.5 & 2.3 & 2.3 & 2.9 & 2.7 & 3.2 & 2.7 & 4.11 \\
\hline
\end{tabular}

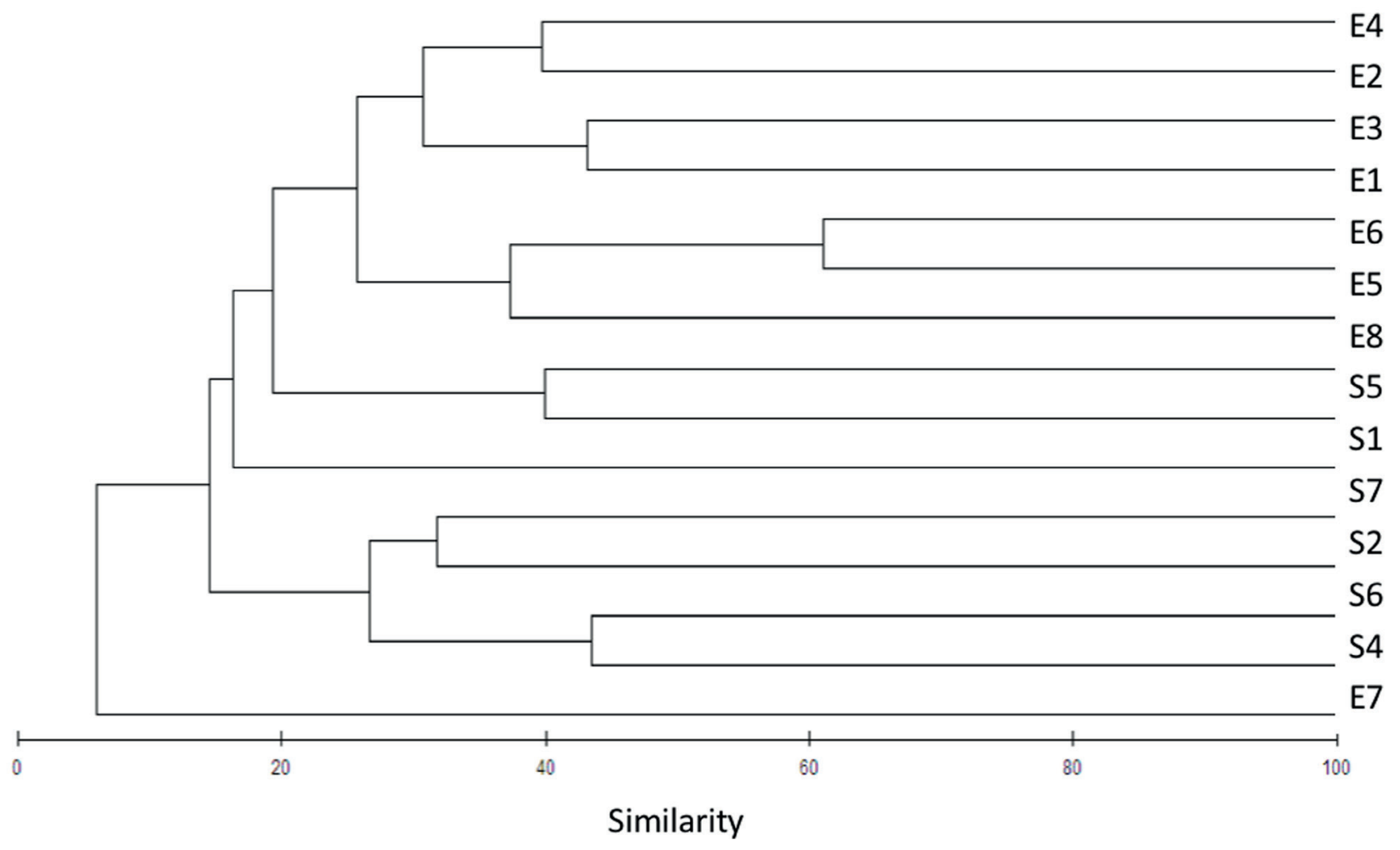

Figure 1. Dendrogram of similarity between the compositions of the bryophytes communities in the campo rupestre areas in the habitats exposed E1-E8 and shaded S1- S7, within Chapada Diamantina, Bahia, Brazil.

areas, most of which formed a cluster. Even geographically close areas exhibited low levels of similarity. Richness was significantly different among the studied areas (T-test $=\mathrm{p}$ $<0.05)$. Seventy-eight species occurred in shaded areas, with 57 being exclusive to this type of habitat, whereas 52 species occurred in exposed areas, with 29 being exclusive; twenty three species were common to both types of habitat.

\section{Frequency of species}

There was a predominance of rare species $(70 \%)$ followed by occasional species $(23 \%)$ and frequent species (7\%). Frequent species were well distributed among the study areas while other species were restricted to a small number of areas or even a single area. Among the frequent species, the following stood out: Campylopus pilifer (49 occurrences/9 areas), Syrrhopodon prolifer var. prolifer (34/9), Schlotheimia rugifolia (25/8), Polytrichum juniperinum (15/3), Campylopus arctocarpus (14/8),
Campylopus julaceus (13/7) and Octoblepharum albidum (12/5). Among the occasional species were Sphagnum palustre (9/5), Lepidozia cupressina (9/3) and Leucobryum martianum (9/3), Anoplolejeunea conferta (9/2), Kurzia capillaris (8/5) and Rhacocarpus purpurascens (6/1). Among the rare species, the following stood out: Anastrophyllum piligerum (1/1), Calymperes palisotii (1/1), Calypogeia laxa (1/1), Neesioscyphus homophyllus (1/1), Wijkia flagellifera (1/1), Lophocolea mandonii (1/1), Campylopus fragilis (2/1), Micropterygium campanense (2/2), Sphagnum oxyphyllum $(2 / 2)$, Odontoschisma brasiliense (4/2).

\section{Substrate colonization}

Four substrates were colonized within the study areas, and corresponded to the following communities: rupicolous, corticicolous, terricolous and epixilous. The rupicolous community predominated in the study ( $86 \%$ of the total number of species), with 70 species being exclusive to this 
substrate ( $66 \%$ of the total number of species). Nineteen species $(18 \%)$ were terricolous with only two exclusive species (1.8\%); fifteen species (14\%) were corticicolous, with nine being exclusive ( $8.4 \%)$; and only six species (5.6 $\%)$ were epixilous, three of which were exclusive to this substrate $(2.8 \%)$. Shaded areas had a greater number of colonized substrates and more species colonizing more than one type of substrate than the exposed areas.

\section{Life and growth forms}

Five life forms were found among the bryophyte flora of Chapada Diamantina, with a predominance of turf (49 spp.), which was the life form of more than $50 \%$ of the species occurred in 10 of the 15 study areas. The weft form (36 spp.) occurred in all areas, likewise the turf form, both corresponding to $78 \%$ of the species. The thalloid form (five spp.) was less frequent. In relation to growth forms, we found 10.4 species on average of acrocarpous mosses and four of pleurocarpous mosses per area. Cladocarpous mosses were also well represented, although they were members of only two families, Orthotrichaceae and Rhizogoniaceae.

\section{Discussion}

The bryophyte flora found in the campos rupestres of Chapada Diamantina was represented mostly by mosses, particularly of the families Leucobryaceae, Sematophyllaceae, Orthotrichaceae and Sphagnaceae. Liverworts were represented by the family Lepidoziaceae. Bryophyte species richness and composition varied widely among the study areas, similar to what has been reported for vascular plants of campos rupestres (Conceição \& Giulietti 2002; Zappi et al. 2003; Conceição \& Pirani 2005; 2007; Conceição et al. 2005). The varied species composition is due to the variety of habitats, determined mainly by relief and proximity to a water source (Alves \& Kolbek 1993; Conceição \& Pirani 2005).

The heterogeneity of the selected habitats translated into distinct bryophyte communities in terms of both composition and life forms. Higher diversity and a greater number of rare species were observed in areas with at higher elevations and with less anthropic activity (areas that are difficult access and thus have low traffic), regardless of the type of habitat investigated.

Large patches of long and dense turfs of Sphagnum spp. were predominant in rock walls of shaded areas, located beneath or close to waterfalls where they are constantly splashed with water. Rock crack communities associated with waterfalls were represented mostly by pleurocarpous mosses of the genera Callicostella, Thamniopsis and Sematophyllum and by leafy liverworts of the genera Micropterygium, Lophocholea and Odontoschisma. Nevertheless, acrocarpous mosses, such as Philonotis, Campylopus and Syrrhopodon, were also present. In general, pleurocarpous mosses and liverworts are adapted to shaded conditions (Richards 1984; Gradstein \& Pócs 1989; Glime 2007). The variety of desiccation tolerance adaptations exhibited by bryophytes is conditioned to the variety of habitats they colonize (Proctor et al. 2007).

The collective bryophyte community of the exposed habitats was represented mainly by acrocarpous mosses of the genera Campylopus and Polytrichum, which have morphological adaptations to withstand intense luminosity and desiccation. Representatives of these genera have lamellae, which possess cells containing chlorophyll aligned in a palisade arrangement on the ventral surface of leaves in such a manner that they are protected from damage caused by strong and prolonged incidence of UV radiation on the chlorophyll. Some species, such as Polytrichum juniperinum, also have extended leaf margins that prevent rapid desiccation, and some species of Campylopus have hyaline hair-point apexes, which give them a grayish appearance and contribute to the reflection of intense luminosity (Mägdefrau 1982; Glime 2007). Species of the genus Polytrichum possess a leaf movement mechanism that responds to environmental humidity by opening the leaves in the presence of water and closing them in dry conditions (Glime 2007). These species have well developed hydroids and are, therefore, endohydric (Schofield 1985; Glime 2007). This type of specialization is associated with humid substrates, and thus, reflects the presence of humidity, such as the misty conditions typical of highelevation environments where air humidity is retained in the shallow soils of campos rupestres.

The high degree of variation in species composition among the study areas, and even over short geographic distances, corroborates the results found for vascular plants in campos rupestres (Conceição \& Giulietti 2002; Zappi et al. 2003; Conceição \& Pirani 2005; 2007; Conceição et al. 2005). However, unlike vascular plants, which exhibit similar species richness among different areas (Conceição \& Pirani 2007), bryophyte richness and diversity differ significantly among the study areas. The low degree of similarity among study areas probably reflects differences in habitat related to the degree of steepness of the relief, quantity and size of rock blocks, presence and size of rock cracks, proportion of exposed rock and wind exposure.

The predominance of the rupicolous community over the others is obviously due to the great availability of rock surfaces. This contrasts with tropical forest environments, where corticicolous bryophytes predominate (Richards 1984; 1988; Gradstein \& Pócs 1989). In the present study, the corticicolous community was generally associated with the stems of herbs and shrubs in exposed areas and with trees in the shaded areas (S6 and S7).

The appearance of bryophytes (life and growth form) as they are found in nature, is clearly influenced by abiotic factors, particularly water and light availability. The bryophyte flora of different vegetation communities and 
habitats develop diversified physiognomies in part due to these life forms, which are of major importance for several vital processes, but particularly water economy (Mägdefrau 1982; Proctor 1982; Bates 1998).

The predominance of turf over other life forms in exposed areas, where plants are subjected to stronger insolation, as well as bryophytes mainly occurring on rock and sandy soils, can be interpreted as evidence of the responses of the bryophytes to the environmental conditions in the studied habitats of the campos rupestres of Chapada Diamantina. This type of life form is known to be tolerant of these conditions, contributes to minimizing water loss of the assemblage of individuals (Mägdefrau 1982), and was exhibited by species of Campylopus, Polytrichum, Schlotheimia, Macromitrium and Syrrhopodon. Turf also occurred in shaded areas, as represented by the genus Sphagnum, which forms long turfs in humid environments (Mägdefrau 1982; Bates 1998). The weft form, typical of humid to more or less humid environments (Mägdefrau 1982; Bates 1998; Glime 2007), was found in larger proportions in shaded areas and was represented by species of Lepidozia, Micropterygium, Bazzania and Odontoschisma. The pendant form was recorded in only one shaded area. This form is typical of humid forests (Gradstein \& Pócs 1989) and its occurrence in the present study is explained by the presence of an arboreal stratum and humidity from mist at the collection site, which was located on a hilltop at 1,290 m.a.s.l.

The high number of rare species, whether classified as restricted (occurring in one or a few areas) or of low frequency (few occurrences), and the high rate of exclusive species ( $79 \%$ of the species were restricted to only one of the investigated habitats) provide evidence of the high degree of heterogeneity of bryophyte communities in the campos rupestres of Chapada Diamantina. Furthermore, these data show the importance of habitat heterogeneity for high diversity, a characteristic also reported for vascular plants (Giulietti \& Pirani 1988; Alves \& Kolbek 1993; Harley 1995a; Giulietti et al. 1996; Conceição \& Giulietti 2002; Conceição et al. 2005; Conceição \& Pirani 2005; 2007).

Some rare species, such as Anastrophyllum piligerum, Lophocolea mandonii, Neesioscyphus homophyllus and Odontochisma brasiliense, which were found inhabiting some study areas that were located within conservation units, have been considered endangered by red lists in the states of Minas Gerais and Rio de Janeiro (Costa et al. 2005; 2006; Costa \& Santos 2009). This finding serves as a warning about the importance and need to preserve the flora of campos rupestres in Chapada Diamantina.

\section{Acknowledgements}

We thank CNPq for the doctoral scholarship granted to the first author, FAPESB for financial support (APR 0066/2007) and IBAMA for the license to collect botanical material. The Departamento de Ciências Biológicas -
Universidade Estadual de Feira de Santana provided infrastructure essential for the development of this research.

\section{References}

Agritempo. 2010. Agritempo site. http://www.agritempo.gov.br. 03 Mai 2010.

Allen B. 2002. Moss flora of Central America - Part 2. EncalyptaceaeOrthotrichaceae. Vol. 90. St. Louis, Missouri Botanical Garden Press.

Alves RJV, Kolbek J. 1993. Penumbral rock communities in campo rupestre sites in Brazil. Journal of Vegetation Science 4: 357-366.

Ayres M, Ayres JM, Ayres DL, Santos AS. 2000. BioEstat 2.0: aplicações estatísticas nas áreas das ciências biológicas e médicas. Belém, Sociedade Civil Mamirauá.

Ballejos J, Bastos CJP. 2009a. Musgos Pleurocárpicos do Parque Estadual das Sete Passagens, Miguel Calmon, Bahia, Brasil. Hoehnea 36: 479-495.

Ballejos J, Bastos CJP. 2009b. Orthotrichaceae e Rhizogoniaceae (Bryophyta - Bryopsida) do Parque Estadual das Sete Passagens, Bahia, Brasil. Rodriguésia 60: 723-733.

Bastos CJP, Yano O, Vilas Bôas-Bastos SB. 2000. Briófitas de Campos rupestres da Chapada Diamantina, Estado da Bahia, Brasil. Revista Brasileira de Botânica 23: 357-368.

Bates JW. 1982. Quantitative Approaches in Bryophyte Ecology. In: Smith AJE. (ed.). Bryophyte Ecology. London, Chapman and Hall Ltd. p. 1-44.

Bates JW. 1998. Is 'life form' a useful concept in bryophyte ecology? Oikos 82: 223-237.

Buck WR. 1998. Pleurocarpous Mosses of the West Indies. Memoirs of The New York Botanical Garden 1: 1-401.

Chao A. 1984. Nonparametric estimation of the numbers of classes in a population. Scandinavian Journal of Statistics 11: 265-270.

Clarke KR, Warwick RM. 1994. Chance in marine communities: an approach to statistical analysis and interpretation. Bournemouth, Bourne Press.

Conceição AA. 2000. Alerta para a conservação da biota na Chapada Diamantina. Revista Ciência Hoje 27: 54-56.

Conceição AA. 2008. Campo Rupestre e o Morro do Pai Inácio. In: Funch LS, Funch RR, Queiroz LP. (eds.) Serra do Sincorá: Parque Nacional da Chapada Diamantina. Feira de Santana, Radami. p. 49-58.

Conceição AA, Giulietti AM. 2002. Composição florística e aspectos estruturais de campo rupestre em dois platôs do Morro do Pai Inácio, Chapada Diamantina, Bahia, Brasil. Hoehnea 29: 37-48.

Conceição AA, Pirani JR. 2005. Delimitação de habitats em campos rupestres na Chapada Diamantina, Bahia: substratos, composição florística e aspectos estruturais. Boletim de Botânica da Universidade de São Paulo 23: 85-111.

Conceição AA, Pirani JR. 2007. Diversidade em quatro áreas de campos rupestres na Chapada Diamantina, Bahia, Brasil: Espécies distintas, mas riquezas similares. Rodriguésia 58: 193-206.

Conceição AA, Rapini A, Pirani JR, et al. 2005. In: Juncá FA, Funch L, Rocha W. (eds.) Biodiversidade e conservação da Chapada Diamantina. Brasília, Ministério do Meio Ambiente. p. 153-180.

Costa DP, Imbassahy CAA, Silva VPAV. 2005. Diversidade e importância das espécies de briófitas na conservação dos ecossistemas do estado do Rio de Janeiro. Rodriguésia 56: 13-49.

Costa DP, Pôrto KC, Starling F, Santos, ND, Yano O. 2006. Briófitas. In: Drummond GM, Martins CS, Mendonça MP. (eds.) Revisão das listas das espécies da flora e fauna ameaçadas de extinção do estado de Minas Gerais. Relatório Final. Belo Horizonte, Fundação Biodiversitas. p. 42-43.

Costa DP, Santos ND. 2009. Conservação de hepáticas na Mata Atlântica do sudeste do Brasil: uma análise regional no estado do Rio de Janeiro. Acta Botanica Brasilica 23: 913-922.

Cowell RK. 2006. EstimateS: Statistical estimation of species richness and shared species from samples. Version 8.0.: http://purl.oclc.org/ estimates.

Crandall-Stotler B, Stotler RE, Long DG. 2009. Morphology and classification of the Marchantiophyta. In: Goffinet B, Shaw AJ. (eds.) Bryophyte biology. 2nd. edn. Cambridge, Cambridge University Press. p. 1-54. 
Crum H. 1984. North American Flora. Sphagnopsida. Sphagnaceae. The New York Botanical Garden 11:1-180

Frahm JP. 1991. Dicranaceae: Campylopodioideae, Paraleucobryoideae. Flora Neotropica Monograph 54: 1-237.

Frahm JP. 1994. The ecology of epiphytic bryophytes on Mt. Kahuzi (Zaire). Tropical Bryology 9: 137-151.

Frahm JP, Gradstein SR. 1991. An altitudinal zonation of tropical rain forests using bryophytes. Journal of Biogeography 18: 669-678.

Fulford M. 1963. Manual of Hepaticae of Latin America. Part I. Memories of The New York Botanical Garden 11: 1-172.

Fulford M. 1966. Manual of Hepaticae of Latin America. Part II. Memories of The New York Botanical Garden 11: 173-276.

Fulford M. 1968. Manual of Hepaticae of Latin America. Part III. Memories of The New York Botanical Garden 11: 277-392.

Giulietti AM, Queiroz LP, Harley RM. 1996. Vegetação e flora da Chapada Diamantina, Bahia. In: Anais da 4 Reunião Especial da SBPC, "Semiárido: no terceiro milênio, ainda um desafio". Feira de Santana, Universidade Estadual de Feira de Santana. p. 144-156.

Giulietti AM, Pirani JR. 1988. Paterns of geographic distribution of some plant species from the Espinhaço Range, Minas Gerais and Bahia, Brazil. In: Vanzolini PE, Heyer WR. Proceedings of a workshop on neotropical distribution patterns. Rio de Janeiro, Academia Brasileira de Ciências. p. 39-69.

Glime JM. 2007. Bryophyte ecology. Michigan, Ebook sponsored by Michigan Technological University and the International Association of Bryologists. http://www.bryoecol.mtu.edu/. 10 Jan. 2010.

Goffinet B, Buck WR, Shaw AJ. 2009. Morphology, anatomy, and classification of the Bryophyta. In: Goffinet B, Shaw AJ. (eds.) Bryophyte biology. 2nd. edn. Cambridge, Cambridge University Press. p. 55-138.

Gradstein SR, Churchill SP, Salazar-Allen N. 2001. Guide to the Bryophytes of Tropical America. Memoirs of the New York Botanical Garden 86: 1-577.

Gradstein SR, Costa DP. 2003. The Hepaticae and Anthocerotae of Brazil. Memoirs of the New York Botanical Garden 87: 1-336.

Gradstein SR, Pócs T. 1989. Bryophytes. In: Lieth H, Werger MJA. (eds.) Tropical rain forest ecosystems. Amsterdan, Elsevier. p. 311-325.

Harley RM. 1995a. Introduction. In: Stannard BL. (ed.) Flora of the Pico das Almas, Chapada Diamantina - Bahia, Brazil. Kew, Royal Botanic Garden. p. 1-40.

Harley RM. 1995b. Bryophyta. In: Stannard BL. (ed.) Flora of the Pico das Almas. Chapada Diamantina - Bahia, Brazil. Kew, Royal Botanic Garden. p. 803-812.

IBGE. 2004. Mapa de Biomas do Brasil. Brasília, Ministério do Planejamento, Orçamento e Gestão.

Mägdefrau K. 1982. Life-forms of bryophytes. In: Smith AJE. (ed.) Bryophyte ecology. Cambridge, Cambridge University Press. p. 45-58

Nolasco MC, Lima CCU, Rocha WF, Rêgo MJM. 2008. Aspectos físicos da Serra do Sincorá, Chapada Diamantina, Bahia. In: Funch LS, Funch RR, Queiroz LP. (eds.) Serra do Sincorá: Parque Nacional da Chapada Diamantina. Feira de Santana, Radami. p. 17-33.

Proctor MCF. 1982. Physiological ecology: water relations, light and temperature responses, carbon balance. In: Smith AJE. (ed.) Bryophyte ecology. London, Chapman \& Hall. p. 333-381.

Proctor MCF, Oliver MJ, Wood AJ, et al. 2007. Desiccation-tolerance in bryophytes: a review. The Bryologist 110: 595-621.

Reese WD. 1993. Calymperaceae. Flora Neotropica Monograph 58: 1-102. Richards WP. 1984. The ecology of tropical forest bryophytes. In: Schuster RM. (ed.) New Manual of Bryology. Vol. 2. Nichinan, Hattori Botanical Laboratory. p. 1233-1270.

Richards WP. 1988. Tropical forest bryophytes. Synusiae and strategies. The Journal Hattori Botanical Laboratory 64: 1-4.

Rocha WJSF, Chaves JM, Rocha CC, Funch L, Juncá FA. 2005. Avaliação ecológica rápida da Chapada Diamantina. In: Juncá FA, Funch L, Rocha W. (eds.) Biodiversidade e conservação da Chapada Diamantina. Brasília: Ministério do Meio Ambiente. p. 29-45.

Schofield WB. 1985. Introduction to Bryology. New York, MacMillan Publishing.

SEI - Superintendência de Estudos Econômicos e Sociais da Bahia. 2003. Fonte dos dados pluviométricos. http://www.sei.ba.gov.br. 01 Feb. 2010.

Sharp AJ, Crum H, Eckel P. 1994. The moss flora of Mexico. Memoirs of The New York Botanical Garden 69: 1-1113.

Thiers B. 2017 [continuously updated]. Index Herbariorum: A global directory of public herbaria and associated staff. New York, New York Botanical Garden's Virtual Herbarium.

Valente EB, Porto KC, Bastos CJP. 2011. Checklist of Bryophytes of Chapada Diamantina, Bahia, Brazil. Boletim do Instituto de Botânica 21: 111-124

Valente EB, Porto KC, Bastos CJP. 2013a. Species richness and distribution of bryophytes within different phytophysiognomies in the Chapada Diamantina region of Brazil. Acta Botanica Brasilica 27: 294-310.

Valente EB, Porto KC, Bastos CJP. 2013b. Diversity and distribution of the bryophyte flora in montane forests in the Chapada Diamantina region of Brazil. Acta Botanica Brasilica 27: 506-518.

Vanderpoorten A, Goffinet B. 2009. Introduction to Bryophytes. Cambridge, Cambridge University Press.

Vaz-Imbassahy TF, Costa DP. 2008. The Pilotrichaceae (Hookeriales) of Rio de Janeiro, Brazil. The Bryologist 111: 551-575.

Velloso AL, Sampaio EVSB, Pareyn FGC. 2002. Ecorregiões propostas para o Bioma caatinga. Recife, Associação Plantas do Nordeste, Instituto de Conservação Ambiental, The Nature Conservancy do Brasil.

Yano O. 1987. Briófitas. In: Giulietti AM, Menezes NL, Pirani JR, Meguro M, Wanderley MGL. (eds.) Flora da Serra do Cipó, Minas Gerais: caracterização e lista de espécies Boletim de Botânica da Universidade de São Paulo 9: 11-12.

Yano O, Peralta DF. 2009. Flora de Grão-Mogol, Minas Gerais: Briófitas (Bryophyta e Marchantiophyta). Boletim de Botânica da Universidade de São Paulo 27: 1-26.

Yano O, Pirani JR, Santos DP. 1985. O gênero Sphagnum (Bryopsida) nas regiões Sul e Sudeste do Brasil. Revista Brasileira de Botânica 8: 55-80.

Zappi DC, Lucas E, Stannard BL, et al. 2003. Lista das plantas vasculares de Catolés, Chapada Diamantina, Bahia, Brasil. Boletim de Botânica da Universidade de São Paulo 21: 345-398. 